\title{
Theoretical Study of Acoustic Circular Arrays With Tangential Pressure Gradient Sensors
}

\author{
Falk-Martin Hoffmann and Filippo Maria Fazi
}

July 31, 2015

\begin{abstract}
Microphone arrays as a means of sound field acquisition have been the topic of extensive research for more than eight decades now. A number of designs have been suggested, each trying to overcome difficulties that are inherent to either the decomposition of the sound field, the transducers in use or the presence of the array itself. This work presents a theoretical analysis of circular microphone arrays that do not measure the sound pressure but the component of its gradient that is tangential to a given boundary. Its performance is compared to that of a conventional pressure sensor array as a benchmark. The focus of the analysis and subsequent assessment lies on spatial aliasing and performance in the presence of noise.
\end{abstract}

\section{Introduction}

The general idea behind microphone arrays is to record sound not only at one point in space, but to capture information about the sound field, such as the direction of travel of incoming wave fronts. Although it is not necessarily the main objective, one ambitious application of microphone arrays is to make a recording of the entire sound field. While in theory this goal can be achieved easily [1], there are various limitations in practice that will degrade the accuracy of the results. Examples of limiting factors are acoustic transducer noise, impractical requirements on the measurement resolution and assumptions on the sound field that cannot be satisfied in practice. Therefore, the focus of the latest research has been on finding means and ways to overcome those limitations.

The field of array technology is vast and certainly not limited to acoustical applications. For example, Van Veen and Buckley presented a general work on array technologies for beamforming applications in wave fields [2], among which are e.g. antennas, sonar systems and microphone arrays. 
Krim and Viberg presented beamforming with sensor arrays as a problem in the field of signal processing, based on a wave propagation model [3] and considering different array geometries. Even though not conceptually limited, this work focuses on airborne wave fields and circular (or cylindrical) arrays.

The earliest approach to a pressure sensor array in the field of airborne acoustics that was applied to make recordings for stereophonic purposes was presented by A. Blumlein [4,5], which had later been modified into using two coincident pressure gradient (figure-of-eight) microphone capsules (also referred to as a "Blumlein-pair"). This recording technology is still used by today's recording engineers, yet it only allows for a two dimensional analysis of the sound field. The first approach to achieve a three dimensional analysis was based on decomposition of the sound field by means of spherical harmonics and was presented by Craven and Gerzon [6], thereby founding the ambisonics technique. The ambisonics approach and also the Higher Order Ambisonics (HOA) approach involve the representation of a sound field as a weighted series of elementary functions in the spatial domain. These functions are determined by the underlying spherical coordinate system, leading to an infinite set of both radial (Bessel- and Hankel-functions) and angular (spherical harmonics) functions. Their form is independent of the sound field itself, ergo the individual weights of the series are sufficient information to know the sound field in its entirety. This is similar to the Fourier analysis presented in [1]. However, it has proven itself difficult to find reliable ways to obtain these weights from measurements.

The general approach of preceding works [7-10] relies on the exploitation of the orthogonality relation of the underlying basis functions and on its application to the array's measured data. One variation of this approach was presented by Rafaely and Park [11,12], who proposed to decompose the sound field into plane waves, which however still involves the exploitation of the orthogonality relation of spherical harmonics. The latter plays a major role in many microphone array designs, but in order for these relations to hold without exception, the sound field must be observed at every single point on the observation boundary. This is so far not feasible in practice, since the number of observation points would need to be infinite. The practical solution is to sample the sound field on that boundary instead. Sampling is however bound to cause aliasing if the observed object's complexity is not within the limitations imposed by the sampling scheme. This is already well known in the field of digitalisation of time domain signals. A thorough analysis of aliasing phenomena in spherical arrays is presented in references $[8,9,13]$. However, aliasing still remains one of the most prominent problems in microphone array design.

Rafaely also identified noise of the array's sensors and inaccuracies in their positioning as significant 
sources of error, and presented a detailed analysis of these issues in [8]. Supporting these results, Poletti has shown that noise induced by the capsules of the array is a problem, especially at low frequencies, because the recovery of the information describing the sound field from the array's observation requires considerably high gain factors for higher order modes. He showed that, for a spherical array with pressure sensors on a rigid sphere, the presence of noise may already pose a fundamental problem for the recovery of the fourth order mode, even with a large dynamic range of $120 \mathrm{~dB}$ for each capsule [14].

Fazi and Nelson presented the theory and analysis of the problem of nonuniqueness [15], as it occurs in the context of sound field acquisition and reproduction, significantly affecting the design of microphone arrays.

For applications in which it is sufficient to consider the sound field in two dimensions only, circular arrays have been investigated as an alternative to spherical arrays. One such application would be a teleconference scenario where one part of the participants is situated at a table and the array serves to separate the individual speakers into individual audio signals. Another example is that of a humanoid robot that needs to distinguish between people addressing him from different directions, so it can then turn towards them. Meyer presented a work on beamforming in combination with circular microphone arrays mounted on spherical objects and already considered the use of pressure sensors as well as dipole sensors [16]. Teutsch and Kellermann have investigated the theory and practicability of a circular array fitted into a cylindrical baffle for source detection and localisation purposes [17]. Kleider et al. [18] investigated the aliasing behaviour of circular arrays based on a two dimensional analysis of the sound field, while Meyer and Elko [19] used a circular array to achieve modal beamforming, still assuming a spherical (three dimensional) sound field model. Poletti [20] investigated the performance of circular arrays w.r.t. noise and transducer variability.

Ever since Blumlein proposed the recording technique based on pressure gradient sensors, the choice of sensors used in microphone arrays was mainly that of pressure sensors. Meyer investigated the use of dipole sensors in a circular array arranged on the equator of a sphere [16]. His work considered radially aligned dipoles as well as dipoles aligned in the circumferential direction. Poletti has considered the application of directional sensors in an open sphere design pointing radially outward, and he found that the problem of nonuniqueness as it occurs with pressure sensors can be overcome [14]. Another more recent design based on differential sensors was presented by Craven, Law and Travis, who proposed a spherical array based on tangential velocity sensors [21]. They found that such a design allows for a reduced effect of noise at lower frequencies. As theirs was a conceptual study, a theoretical analysis 
of the design has so far not been presented.

On the background of the work of Craven, Law and Travis, it should be noted that, in homogeneous sound field conditions, the pressure gradient relates to the velocity by Euler's equation. On the surface of a rigid object, however, this relation is no longer valid and needs to be replaced by a set of equations describing the laminar sound field behaviour within the acoustic boundary layer. These can be derived from the Navier-Stokes equations. A detailed analysis of this is not within the scope of this work, but should be mentioned for the sake of completeness.

In this study, a theoretical analysis of a circular microphone array is presented, where the array is composed of sensors that measure the tangential component of the pressure gradient. An initial study of this design was presented by the authors in reference [22]. This work complements the initial work with further theoretical and numerical in-depth analysis, as well as with an investigation of the noise and aliasing performance and the array's optimal frequency band. In Section 2, a mathematical model of the sound field is presented that is based on the Herglotz Wave Function, modelling a sound field as a superposition of plane waves. The relation between the sound field and the observation of the microphone array is expressed by means of an integral operator. This leads to an inverse problem, which is dealt with in a functional analysis framework. This mathematical approach is not very common in the field of transducer array research, but it is a very neat and effective tool to perform the type of analysis this work presents. It has been applied in previous work by Colton and Kress [23] and by Fazi [24]. In Section 3, the presented array model is discretised and a thorough analysis of the array's aliasing behaviour is undertaken. Section 4 provides a simulation based study of the array's performance for the measurement of the approximated sound field of a single plane wave, comparing the recovered sound field information to the theoretical results, evaluating the overall error of the recovery and discussing the significance of aliasing and transducer noise as a problem. The final section summarises the findings of this work and gives a brief overview on upcoming research.

\section{Model of the Sound Field}

For the subsequent analysis, a mathematical model describing the pressure of an arbitrary sound field within a given region of space (or area, in the two-dimensional case) $\Lambda$ is needed. The model relies on the assumption that the measured sound field can be represented as the superposition of an infinite number of plane waves. This model is particularly useful when considering a limited number 

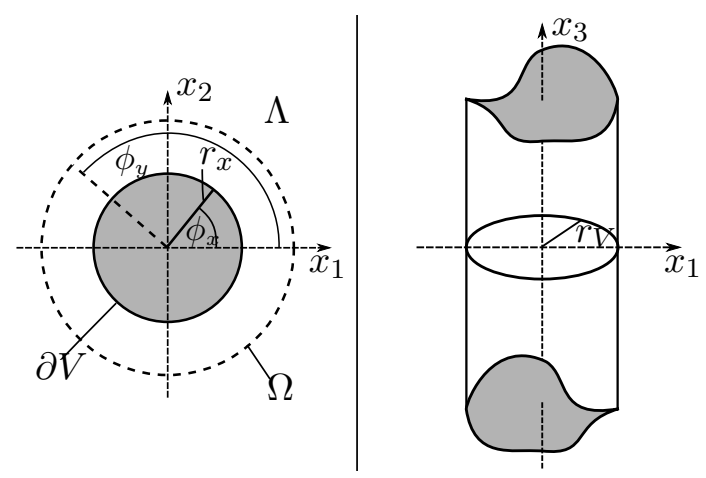

Figure 1: (left) Schematic description of the volumes and boundaries involved in the sound field model, (right) schematic description of an infinite cylinder of radius $r_{V}$.

of incoming plane waves (see Section 4). When dealing with spherical or cylindrical geometries, it is convenient to represent the plane wave model by means of Fourier expansion. This is the approach chosen in this work.

\subsection{Plane-Wave Decomposition}

The equation

$$
p(\mathbf{x}, \omega)=\int_{\Omega} H(\mathbf{x}, \hat{\mathbf{y}}, \omega) q(\hat{\mathbf{y}}, \omega) d \Omega(\hat{\mathbf{y}}), \mathbf{x} \in \Lambda,
$$

as given in [25], describes the acoustic pressure at an arbitrary position $\mathbf{x}$ as a linear superposition of an infinite number of plane waves travelling into all possible directions $\hat{\mathbf{y}}$, where $\hat{\mathbf{y}}$ is a unitary vector. $\omega=2 \pi f$ denotes the angular frequency corresponding to the acoustic frequency $f$ and $\Omega$ represents the unit sphere or circle in $\mathbb{R}^{3}$ or $\mathbb{R}^{2}$, respectively. The above integral equation is also referred to as the Herglotz Wave Function (HWF) [25] where the Herglotz density (HD) $q(\hat{\mathbf{y}}, \omega)$ describes the complex amplitude of the different plane waves. These are expressed by the kernel $H(\mathbf{x}, \hat{\mathbf{y}}, \omega)=e^{i k \mathbf{x} \cdot \hat{\mathbf{y}}}$, where $i=\sqrt{-1}$ is the imaginary unit and $k$ is the acoustic wave number ${ }^{1}$. In principle, equation (1) is valid for $\Lambda=\mathbb{R}^{3}$ (or $\Lambda=\mathbb{R}^{2}$ ), provided that the sound field satisfies the homogeneous wave equation in that domain. In practice, this equation is often used to represent a sound field that satisfies the homogeneous wave equation only in a bounded domain $\Lambda$.

This representation is used as a foundation for the theory of the baseline microphone array design, using pressure sensors only. For the sake of brevity, the argument $\omega$ is omitted in all equations used hereunder, since all calculations are derived for a single frequency $\omega$.

\footnotetext{
${ }^{1}$ Note that the Herglotz Wave Function as given in [25] uses a complex conjugate kernel $e^{-i k \mathbf{x} \cdot \hat{\mathbf{y}}}$ instead, since Fazi et al. define $\hat{\mathbf{y}}$ as the direction of arrival, instead of the direction of propagation.
} 
In the subsequent analysis, all considerations are limited to a two-dimensional scenario, assuming that the sound field is constant along the $x_{3}$-axis with

$$
p\left(x_{1}, x_{2}, x_{3}\right)=p\left(x_{1}, x_{2}\right) .
$$

This is a common means of simplification when a height-invariant sound field is observed on the boundary $\delta V$ of an infinitely long cylinder extending along the $x_{3}$-axis, as depicted in Fig. 1 . The assumption given in equation (2) then allows for another simplification from cylindrical coordinates to polar coordinates, so that

$$
\begin{aligned}
& x_{1}=r_{x} \cdot \cos \phi_{x}, \text { and } \\
& x_{2}=r_{x} \cdot \sin \phi_{x},
\end{aligned}
$$

where $\phi_{x}=\arctan \frac{x_{2}}{x_{1}}$ is the polar angle of a vector $\mathbf{x}=\left[x_{1}, x_{2}\right]^{T}$.

Since this study aims at an array design observing the tangential component $g$ of the pressure gradient, an expression similar to (1), relating the HD and the tangential pressure gradient (TPG) component, needs to be found. With the assumptions made in the previous paragraph, the latter is defined by the scalar product

$$
\left.g(\mathbf{x})\right|_{\mathbf{x} \in \partial V}=\nabla p(\mathbf{x}) \cdot \mathbf{b}(\mathbf{x})
$$

$\partial V$ denotes the boundary of the microphone array which embeds all the observation points ${ }^{2}$ or sensors, respectively. $\mathbf{b}(\mathbf{x})$ represents the unit vector pointing in the direction tangential to $\partial V$ at the observation point $\mathbf{x}$. It is important to realise that, depending on the chosen coordinate system and on the shape of $\partial V$, the analytical expression for $g$ given by (5) may become considerably more complicated.

Let $\partial V$ be a circle of radius $r_{V}$. Using the Jacobi-Anger expansion [23]

$$
e^{i k \mathbf{x} \cdot \hat{\mathbf{y}}}=\sum_{n=-\infty}^{\infty} i^{n} J_{n}\left(k r_{x}\right) e^{i n \phi_{x}} e^{-i n \phi_{y}}
$$

to replace the kernel, equation (1) can be reformulated in polar coordinates, thus obtaining

$$
p\left(r_{x}, \phi_{x}\right)=\sum_{n=-\infty}^{\infty} i^{n} J_{n}\left(k r_{x}\right) e^{i n \phi_{x}} \int_{0}^{2 \pi} e^{-i n \phi_{y}} q\left(\phi_{y}\right) d \phi_{y} .
$$

\footnotetext{
${ }^{2}$ This denomination is compliant with the work of Williams [1], chapter 8, page 258 .
} 
$\phi_{x}$ and $\phi_{y}$ correspond to the polar angles of $\mathbf{x}$ and $\hat{\mathbf{y}}$, respectively. $J_{n}\left(k r_{x}\right)$ denotes the Bessel function of order $n$ and describes the radial component of the sound field. To allow for modelling either free field conditions (FF) or the sound field in the presence of a rigid infinite cylindrical scatterer (CS) of radius $r_{s}$ at the origin, $J_{n}\left(k r_{x}\right)$ needs to be replaced by a more general radial function $R_{n}\left(k r_{x}\right)$. For reasons of brevity, the complex factor $i^{n}$ in (7) is also included in this radial function, so that the latter is defined by

$$
R_{n}\left(k r_{x}\right)=i^{n} \begin{cases}J_{n}\left(k r_{x}\right) & , \mathrm{FF} \\ J_{n}\left(k r_{x}\right)-\frac{J_{n}^{\prime}\left(k r_{s}\right)}{H_{n}^{(1) \prime}\left(k r_{s}\right)} H_{n}^{(1)}\left(k r_{x}\right) & , \mathrm{CS}\end{cases}
$$

where $H_{n}^{(1)}(x)$ denotes the Hankel function of the first kind of order $n$, and $J_{n}^{\prime}\left(k r_{s}\right)$ and $H_{n}^{(1) \prime}\left(k r_{s}\right)$ are the derivatives of the Bessel function and the Hankel function, respectively, evaluated at the boundary of the rigid cylindrical scatterer. The derivation of $R_{n}(x)$ can be found in [1], Section 6.10 . For $r_{s}=r_{V}$, the tangential component of the pressure gradient on $\partial V$ is now a function of $\phi_{x}$ only and is given by [1]

$$
g\left(\phi_{x}\right)=\left.\frac{1}{r_{x}} \frac{\partial p\left(r_{x}, \phi_{x}\right)}{\partial \phi_{x}}\right|_{r_{x}=r_{V}}
$$

Equations (7) and (9) then provide the relation between the HD $q\left(\phi_{y}\right)$ and the pressure on $\partial V$ and its gradient, respectively. In order to obtain $q\left(\phi_{y}\right)$ from the observed pressure $p\left(\phi_{x}\right)$ or from its gradient $g\left(\phi_{x}\right)$, respectively, the corresponding integral equations need to be solved for $q\left(\phi_{y}\right)$.

A common approach $[8,14,26]$ to obtain a solution, based on the observation of the pressure, is to represent the HD by a Fourier series

$$
q\left(\phi_{y}\right)=\sum_{m=-\infty}^{\infty} q_{m} \frac{e^{i m \phi_{y}}}{\sqrt{2 \pi}}
$$

where

$$
q_{m}=\int_{0}^{2 \pi} q\left(\phi_{y}\right) \frac{e^{-i m \phi_{y}}}{\sqrt{2 \pi}} d \phi_{y} .
$$

Replacing $q\left(\phi_{y}\right)$ in $(7)$, replacing $i^{n} J_{n}\left(k r_{x}\right)$ with $R_{n}\left(k r_{x}\right)$ and using the orthogonality relation

$$
\int_{0}^{2 \pi} e^{i m \phi} e^{-i n \phi} d \phi=2 \pi \delta_{m, n}
$$


leads to the expression of the Fourier coefficients

$$
q_{m}=\frac{1}{R_{m}\left(k r_{V}\right)(2 \pi)^{\frac{3}{2}}} \int_{0}^{2 \pi} e^{-i m \phi_{x}} p\left(\phi_{x}\right) d \phi_{x}, \forall m \in \mathbb{Z}
$$

It is important to realise that this approach leads to a unique solution only if $R_{m}\left(k r_{V}\right) \neq 0, \forall m \in \mathbb{Z}, \forall k$. Similarly, as $R_{m}\left(k r_{V}\right)$ can converge to zero for high values of $m$, a solution may exist and be unique, yet be potentially unstable. As shown below, such a solution based only on the observation of the TPG on a boundary $\partial V$ recovering all coefficients $q_{m}, m \in \mathbb{Z}$ cannot be found. This poses a significant problem to the intended array design.

Nevertheless, a derivation similar to that shown in the previous paragraph and based on equation (9) would lead to a very similar solution, however, only for this particular geometry. For the sake of a more general approach, the recovery of $q\left(\phi_{y}\right)$ from $g\left(\phi_{x}\right)$ is derived in the following subsection, using functional analysis as a tool.

\subsection{Analysis of the Integral Operator $G$}

The two quantities of major interest in the given sound field model are the HD $q(\phi)$ and the pressure gradient $g(\phi)$. Both $q(\phi)$ and $g(\phi)$ are assumed to be square-integrable and are considered as elements of an open Hilbert space $A$. Furthermore, it is assumed that $q$ and $g$ can be described through an infinite weighted sum of orthonormal basis functions $a_{n}(\phi) \in A$.

$$
\begin{aligned}
& q(\phi)=\sum_{n=-\infty}^{\infty} q_{n} a_{n}(\phi), q_{n} \in \mathbb{C}, \text { and } \\
& g(\phi)=\sum_{n=-\infty}^{\infty} g_{n} a_{n}(\phi), g_{n} \in \mathbb{C} .
\end{aligned}
$$

Note that, since both functions are elements of $A$, the indices $x$ and $y$ of both $r$ and $\phi$ have been dropped in the following. The operator $H$ is defined by evaluating equation (7) only for $\mathbf{x} \in \partial V$ and by replacing $J_{n}(x)$ by $R_{n}(x)$, yielding

$$
\begin{aligned}
& (H q)(\mathbf{x}):=p\left(\phi, r_{V}\right) \\
& =\sum_{n=-\infty}^{\infty} R_{n}\left(k r_{V}\right) e^{i n \phi} \int_{0}^{2 \pi} e^{-i n \phi^{\prime}} q\left(\phi^{\prime}\right) d \phi^{\prime}, \mathbf{x} \in \partial V
\end{aligned}
$$


with $H: A \rightarrow A$, which is of no further interest in this study. However, replacing $p$ in (9) by (16) leads to the integral equation

$$
g(\phi)=\sum_{n=-\infty}^{\infty} R_{n}\left(k r_{V}\right) \frac{1}{r_{V}} \frac{\partial}{\partial \phi}\left(e^{i n \phi}\right) \int_{0}^{2 \pi} e^{-i n \phi^{\prime}} q\left(\phi^{\prime}\right) d \phi^{\prime},
$$

that is similar to (16). This equation defines a new integral operator $G: A \rightarrow A$ that maps a given HD $q(\phi)$ to the corresponding pressure gradient $g(\phi)$ on $\partial V$ :

$$
\begin{aligned}
& (G q)(\mathbf{x}):=g(\phi) \\
& =\sum_{n=-\infty}^{\infty} R_{n}\left(k r_{V}\right) \frac{i n}{r_{V}} e^{i n \phi} \int_{0}^{2 \pi} e^{-i n \phi^{\prime}} q\left(\phi^{\prime}\right) d \phi^{\prime}, \mathbf{x} \in \partial V
\end{aligned}
$$

This equation perfectly describes what a TPG sensor located at $\phi$ observes in a sound field that is defined by the HD $q(\phi)$. This result was presented before in [22] and is also very similar to what Meyer described in principle for dipole sensors aligned with a circumferential orientation on the equator of a sphere [16].

Aiming at a more general approach to aliasing analysis, the following subsection briefly introduces the eigenvalue decomposition of $G$.

\subsection{Eigenvalue Decomposition (EVD) of $G$}

$G$ has been identified as a mapping operator from a function $q(\phi) \in A$ to a function in $g(\phi) \in A$. For a better understanding of the relation between $q$ and $g, G$ is analysed w.r.t. how the strength of a mode (see below) of $q$ is transmitted to the corresponding mode of $g$.

The eigenvalue decomposition of the operator $G$ is based on the equation

$$
\left(G a_{n}\right)(\phi)=\lambda_{n} a_{n}(\phi)
$$

where $\lambda_{n}$ denotes the eigenvalue associated to the corresponding eigenfunction $a_{n}(\phi)$ of $G$, where the latter is hereafter referred to as a mode. The eigenvalues and eigenfunctions of $G$ for the case under 
consideration are

$$
\begin{aligned}
\lambda_{n} & =2 \pi \frac{i n R_{n}\left(k r_{V}\right)}{r_{V}}, \\
a_{n}(\phi) & =\frac{e^{i n \phi}}{\sqrt{2 \pi}}
\end{aligned}
$$

When comparing equation (18) to the results of the EVD (equations (20) and (21)), it can be assumed that the operator $G$ can be written as follows:

$$
(G q)(\phi)=\sum_{n=-\infty}^{\infty} a_{n}(\phi) \lambda_{n}\left\langle a_{n} \mid q\right\rangle_{\Omega}=g(\phi) .
$$

This expression may be interpreted as the spectral decomposition of the compact operator $G$ [23], where $\langle f \mid g\rangle_{\Omega}=\int_{\Omega} f^{*}(x) g(x) d \Omega(x)$ describes the scalar product of two functions in $A$. This scalar product serves to extract the coefficient $q_{n}$ of the $n$th mode $a_{n}$ as a component of $q$. The extracted mode strength $q_{n}$ is then weighted by the corresponding eigenvalue $\lambda_{n}$ and multiplied with the mode itself again. This formulation allows for the interpretation of the eigenvalues as coupling factors that describe how the mode strength in $q(\phi)$ is transformed to the resulting function $g(\phi)[24]$.

It is evident that not all eigenvalues are non-zero. The most trivial case is that of $\lambda_{0}=0$, which corresponds to the mode $a_{0}(\phi)=\frac{1}{\sqrt{2 \pi}}$. This result implies that a constant

$$
q(\phi)=\kappa \in \mathbb{C},
$$

is an element of the null space $N(G)$ of the operator $G$. This can be easily proven when evaluating equation (18) for a constant $q$. In fact, the latter is still of the form given in (14) and, hence, an element of $A$, where $q_{n}=0, \forall n \neq 0$. Further zero eigenvalues can arise from an open cylinder array design when $R_{n}\left(k r_{V}\right)=0$. The null space of $G$ has a crucial influence on the existence of a unique solution to the inverse problem, as shown in subsection 2.5 .

\subsection{The Eigenvalues $\lambda_{n}$}

A deeper analysis of the eigenvalues provides a theoretical insight into the system's vulnerability to both aliasing and transducer noise. The latter becomes a problem when the observation $g(\phi)$ is heavily amplified for the recovery of a coefficient $q_{n}$, while the former is an inherent product of discretisation. 
The influence of the eigenvalues on the aliasing behaviour is investigated in more detail in Section 3 .

In this section, the noise performance is analysed. First, it is necessary to analyse how the strength $q_{n}$ of a mode $a_{n}(\phi)$ in $q(\phi)$ is transformed into the corresponding mode strength $g_{n}$ in $g(\phi)$. With $g(\phi)=(G q)(\phi)$ and comparing the different factors in equations (22) and (15), it can be seen that the following equation must hold:

$$
g_{n}=\lambda_{n}\left\langle a_{n} \mid q\right\rangle_{\Omega}=\lambda_{n} q_{n} .
$$

As the scalar product simply extracts the strength of the $n$th mode from $q(\phi)$, it is evident that the eigenvalues serve as complex gain factors. When assessing the theoretical performance of microphone arrays, it is more interesting to study the above equation after rearranging it for $q_{n}$. This yields

$$
q_{n}=\frac{1}{\lambda_{n}} g_{n}, \text { for } \lambda_{n} \neq 0
$$

This shows that the desired mode strength $q_{n}$ is calculated by extracting $g_{n}$ from the array observation and then weighting it by $\frac{1}{\lambda_{n}}$. However, for very small $\lambda_{n}$, the fraction in (25) becomes very large. In such a case, when $g_{n}$ happens to be slightly corrupted by measurement noise, the resulting $q_{n}$ is very likely to significantly deviate from the actual value, as the noise component in $g_{n}$ is heavily amplified.

To define if the problem introduced above is of any practical significance, the behaviour of the eigenvalues as a function of frequency needs to be analysed. Fig. 2 shows the graphs of the magnitude of the first $^{3}$ seven non-zero eigenvalues $(n=1 \ldots 7)$ within the audible frequency range. It can be

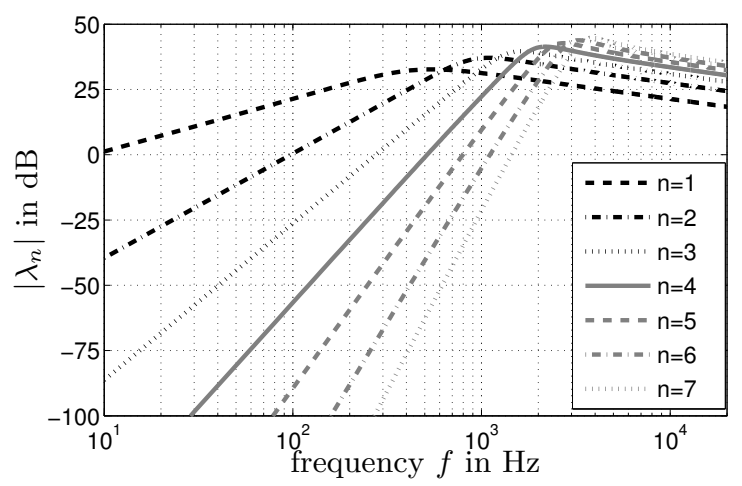

Figure 2: Magnitude of the eigenvalues $\left|\lambda_{n}\right|$ of $G$ for $n=1 \ldots 7, f=10 \ldots 20 \cdot 10^{3} \mathrm{~Hz}$ for an array on a rigid cylindrical structure at the origin with $r_{V}=0.1 \mathrm{~m}$.

observed that the development of eigenvalues of different orders for a TPG array roughly compares to

\footnotetext{
${ }^{3}$ Since the eigenvalues have not been ordered so far, the term 'first' simply refers to the index $n$.
} 
that of the eigenvalues of the operator $H$

$$
\nu_{n}=2 \pi R_{n}\left(k r_{V}\right)
$$

for a conventional pressure sensor array, which is presented in Fig. 3. An analogous plot was already presented by Meyer [16] for a circular array mounted on the equator of a sphere and by Elko and Meyer [7] for the case of spherical arrays. Poletti presented an equivalent plot for a spherical array with radially aligned first order sensors [14].

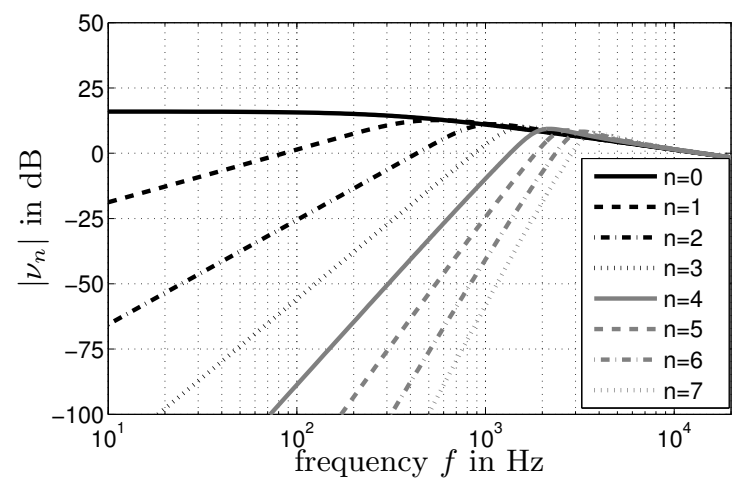

Figure 3: Magnitude of the eigenvalues $\left|\nu_{n}\right|$ of $H$ of a conventional array based on pressure sensors on a rigid cylindrical structure with $r_{V}=0.1 \mathrm{~m}$ for $n=0 \ldots 7, f=10 \ldots 20 \cdot 10^{3} \mathrm{~Hz}$.

Two major differences between the two figures are that, firstly, Fig. 2 does not include $\lambda_{0}$ and, secondly, the additional component $\frac{|n|}{r_{V}}$, which does not affect the shape of the individual graph (in a dB scale) but translates them vertically.

In conclusion, similar to an array using radially outward pointing gradient sensors [14], the design investigated in this work is expected to achieve a better noise performance than arrays with pressure sensors [21]. This is not only the case at low frequencies but overall because of the additional gain component mentioned above. Of course, this statement relies on the assumption that pressure gradient sensors and pressure sensors are of equal quality. However, for $n=7$, the gain that needs to be applied to the measured $g_{7}$ for the recovery of $q_{7}$ is approximately $+25 \mathrm{~dB}$ at $f=1 \mathrm{kHz}$ and $r_{V}=0.1 \mathrm{~m}$ with the TPG sensor array, while it is more than $+50 \mathrm{~dB}$ for the same scenario with a pressure sensor array. This leads to the assumption that a TPG sensor array allows for an increased spatial resolution in scenarios with transducer noise (see Section 4).

While the overall development of the eigenvalues appears to make the array more robust against noise, the additional component $\frac{|n|}{r_{V}}$, which depends linearly on the order $n$, reduces the system's robustness against aliasing. This is further investigated in Section 3. 


\subsection{Solution of the Inverse Problem}

In order to retrieve $q(\phi)$ from the observation of $g$, it is necessary to invert $G$. Considering equation (22) and taking into account that the basis functions are orthonormal, the solution to the inverse problem is

$$
\tilde{q}(\phi)=\sum_{\substack{n=-\infty \\ n \neq 0}}^{\infty} a_{n}(\phi) \frac{1}{\lambda_{n}}\left\langle a_{n} \mid g\right\rangle_{\Omega}=\sum_{\substack{n=-\infty \\ n \neq 0}}^{\infty} a_{n}(\phi) \tilde{q}_{n} .
$$

From equation (27), it is evident why, in order for $\tilde{q}$ to be bounded, the mode $a_{0}(\phi)$ corresponding to the eigenvalue $\lambda_{0}=0$ must be excluded from the solution. As a matter of fact, the mode $a_{0}(\phi)$ is in the null space of the adjoint operator $G^{*}$ and therefore does not satisfy the first Picard condition for the uniqueness of a solution $[23,24]$. Furthermore, considering equation (20) it is also evident why the solution does not exist when

$$
R_{n}\left(k r_{V}\right)=0 .
$$

The limitation implied by $(28)$ is a well-known problem in the field of microphone array research [14,15], which is often overcome in practice by choosing designs based on a rigid array structure.

Another requirement for the solution to be bounded is that the expression

$$
\sum_{\substack{n=-\infty \\ n \neq 0}}^{\infty} \frac{\left|\left\langle a_{n} \mid g\right\rangle_{\Omega}\right|^{2}}{\lambda_{n}^{2}}<\infty
$$

is satisfied, which corresponds to the second condition of Picard's theorem. In theory, this is potentially not satisfied; however, the order truncation discussed in Section 3 ensures that this condition is always satisfied. Finally, the explicit expression of the solution is

$$
\tilde{q}(\phi)=\sum_{\substack{n=-\infty \\ n \neq 0}}^{\infty} e^{i n \phi} \frac{-i r_{V}}{4 \pi^{2} n R_{n}\left(k r_{V}\right)} \int_{0}^{2 \pi} e^{-i n \phi^{\prime}} g\left(\phi^{\prime}\right) d \phi^{\prime}
$$

It follows from (27), (29) and (12) that the series coefficients are given by

$$
\tilde{q}_{n}=\frac{-i r_{V}}{(2 \pi)^{\frac{3}{2}} n R_{n}\left(k r_{V}\right)} \int_{0}^{2 \pi} e^{-i n \phi^{\prime}} g\left(\phi^{\prime}\right) d \phi^{\prime}, \forall n \in \mathbb{Z} \backslash\{0\} .
$$

Equations (29) and (30) have already been presented in [22] but they were derived from a Singular Value Decomposition instead. The result in (30) is very similar to that given in (13). The minor 
difference in the denominator of the fraction is due to the use of the TPG instead of the pressure itself.

The solution presented above leads to a valid HD. However, it is crucial to realise that, since the nullspace of $G$ (see eq. (23)) is non-trivial, the solution $\tilde{q}(\phi)$ recovered from the observation of $g$ may differ from the original HD $q(\phi)$ by an additional constant factor $\alpha$, because any solution

$$
q(\phi)=\tilde{q}(\phi)+\alpha, \alpha \in \mathbb{C}
$$

is also a solution. This must be the case since $\alpha=q_{0}$ (compare eq. (11) for $m=0$ ), which can also be extracted from $q(\phi)$ by exploiting orthogonality (see eq. (12)).

The zero-order mode represents the direct component $q_{0}$ in (14). The physical interpretation of this mode is most easily understood, considering the source-receiver reciprocity, as the sound field generated by a breathing cylinder, i.e. an outgoing or incoming wave with a constant magnitude and phase for all angles at a fixed radius $r_{V}$. This can be easily proven by evaluating $(7)$ for $q(\phi)=q_{0} a_{0}(\phi)=\frac{q_{0}}{\sqrt{2 \pi}}$. As a consequence, the TPG $g$ is not affected by a direct component $q_{0}$ of $q$, which unfortunately implies that $q_{0}$ cannot be recovered from the knowledge of $g$. Moreover, the implicit assumption made in this section, that $q_{0}=0$, imposes a significant restriction to generality.

These findings are rather discouraging, considering the original objective to capture an entire sound field from an array consisting of TPG sensors only. Nevertheless, it is shown below that this problem can be overcome in certain conditions by adding one or more pressure sensors to the array.

\section{Discretisation and Aliasing Analysis}

Similar to the sampling of a time domain signal, the sampling of the pressure gradient on a circle with radius $r_{V}$ leads to spatial aliasing effects, if the sound field contains modes of higher order than those captured by the array. These higher order modes are bound to corrupt the observed modes. An odd number $L$ of TPG sensors distributed uniformly on the circle allows for the recovery of modes $a_{n}(\phi),|n| \leq N$ with

$$
N=\frac{L-1}{2}
$$

The reconstruction of a sound field containing modes of order higher than $N$ is investigated in the following. For reasons of brevity, the argument of the radial functions $R_{n}\left(k r_{V}\right)$ has been omitted 
hereunder.

The integral in (30) is discretised with $L$ sampling points and the equation is then rewritten as

$$
\tilde{q}_{n}=\frac{-i r_{V}}{n R_{n}(2 \pi)^{\frac{3}{2}}} \sum_{l=1}^{L} e^{-i n \Delta \phi l} g(\Delta \phi l) \Delta \phi, \forall n \in \mathbb{Z} \backslash\{0\}
$$

where $\Delta \phi=\frac{2 \pi}{L}$ is the angular spacing between neighbouring sensors.

\subsection{The Aliasing Pattern}

Replacing $g$ by the series expansion given in (15) and using (21) leads to

$$
\tilde{q}_{n}=\frac{1}{\lambda_{n}} \sum_{m=-\infty}^{\infty} g_{m} A_{m, n}
$$

where the factors

$$
A_{m, n}=\frac{1}{L} \sum_{l=1}^{L} e^{-i n \Delta \phi l} e^{i m \Delta \phi l}=\left\{\begin{array}{l}
1, m=n+u L, u \in \mathbb{Z} \\
0, \text { otherwise }
\end{array}\right.
$$

are the elements of a matrix that describes the aliasing pattern of the system. The results in (34) and (35) have already been found in a similar form by Poletti in [20]. Fig. 4 shows the values of (35) evaluated for a circular array with $L=15$ microphones and $n \in[-7, \ldots, 0, \ldots, 7]$ and $m \in$ $[-22, \ldots, 0, \ldots, 22]$. Black cells indicate values of $A_{m, n}$ that are different from zero, hence indicating

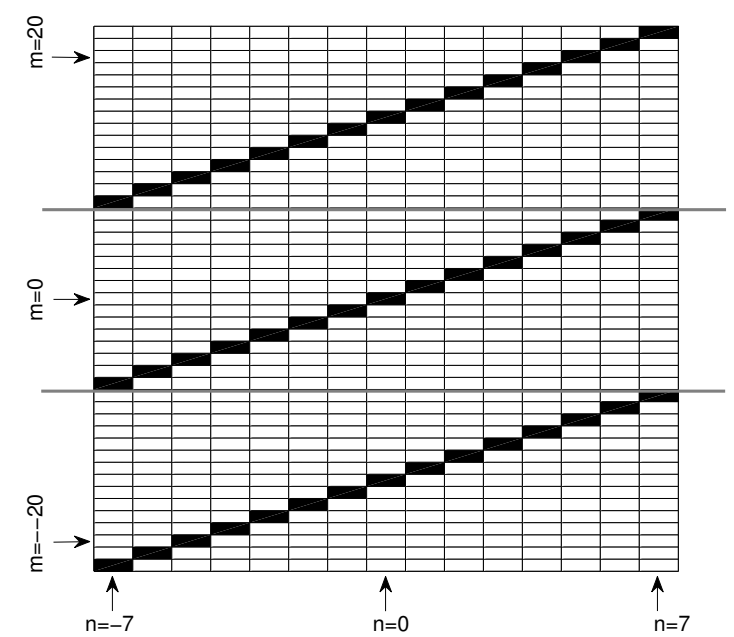

Figure 4: Visualisation of the orthogonality matrix $A_{m, n}$ for a circular array with $L=15$ microphones. Each cell represents whether two modes $a_{m}$ and $a_{n}$ are orthogonal (white cell) or not (black cell), when sampled at $L$ points on a circle. The two light grey lines indicate the mode range $( \pm N)$ of the array. 
a pair of modes that are not orthogonal to each other. As such, Fig. 4 is a good indicator of which modes $n$ recovered by the array are corrupted by modes of order $m$ of the sound field - in other words, aliasing. For example, a recovered coefficient $\tilde{q}_{7}$ of the mode corresponding to $n=7$ is corrupted by aliasing, if the sound field contains modes of order $m=7+u L, u \in \mathbb{Z}$, i.e. $m=-8$ and $m=22$ as given in Fig. 4.

Repeating the same steps as above, starting from equation (13) leads to the same orthogonality matrix $A_{m, n}$ for an array composed of pressure sensors. This shows the aliasing scheme to be the same for both array types.

Using eq. (24) in (33) and exploiting (35) leads to the following equation describing precisely the aliasing behaviour

$$
\tilde{q}_{n}=q_{n}+\sum_{\substack{u=-\infty \\ u \neq 0}}^{\infty} \frac{\lambda_{n+u L}}{\lambda_{n}} q_{n+u L} .
$$

Following mathematical passages similar to those presented above, it can be easily shown that equations (24) and (36) are the same for a pressure sensor array but with different eigenvalues $\nu_{n}$ [24].

The ratio of two eigenvalues $\frac{\lambda_{n+u L}}{\lambda_{n}}$ in (36) has a major effect on the vulnerability to aliasing. The reason for this can be seen from the graphs of the eigenvalues in Fig. 2. The additional factor $|n|$ leads to an amplified contribution of aliased modes on the TPG observation. It can therefore be expected that for the proposed design the aliasing induced at high frequencies by high order modes is worse compared to that of pressure sensor arrays for which the ratio $\left|\frac{\nu_{n}+u L}{\nu_{n}}\right|$ remains close to one (compare to Fig. 3 and the results in [14]). However, since the maximum of $\lambda_{n}$ shifts towards higher frequencies with increasing $n$ (see Fig. 2), this effect is only relevant up to a certain $u_{a}$ where $\frac{\lambda_{n+u_{a} L}}{\lambda_{n}}<1$.

Considering the case of $n=0$, Fig. 4 does not give any information on the aliasing pattern for the 0th order mode since $q_{0}$ cannot be recovered from an array using pressure gradient sensors only. To overcome this limitation, the array needs to be extended by at least one pressure sensor, leading to an overall amount of $L+1$ sensors.

A linear algebra formulation of the mode recovery problem is introduced in the following subsection and then one additional pressure sensor is included into the system. 


\subsection{Mode Recovery using Linear Algebra}

In the following, the objective is to recover modes up to order $N$ from the data acquired with $L$ sensors. For a given HD with limited order $N$ (aliasing free case)

$$
q(\phi)=\sum_{n=-N}^{N} q_{n} a_{n}(\phi),
$$

the result of equation (18) can be rewritten as a function of the coefficients $q_{n}$. Using (15), (21) and (24) yields a solution for the TPG with limited order $N$, given by

$$
g(\phi)=\sum_{n=-N}^{N} \frac{i n}{r_{V}} R_{n} \sqrt{2 \pi} e^{i n \phi} q_{n} .
$$

The expression of (38), when evaluated for all $L$ observation points, leads to a system of linear equations, which can be written using matrix notation:

$$
\mathbf{g}=\left[\begin{array}{c}
g(\Delta \phi \cdot 1) \\
\vdots \\
g(\Delta \phi \cdot L)
\end{array}\right]_{L \times 1}=\mathbf{H J}\left[\begin{array}{c}
q_{-N} \\
\vdots \\
q_{-1} \\
q_{1} \\
\vdots \\
q_{N}
\end{array}\right]_{2 N \times 1}=\mathbf{H J q}
$$

where

$$
\mathbf{J}=\frac{i \sqrt{2 \pi L}}{r_{V}} \cdot \operatorname{diag}\left(-N R_{-N}, \ldots,-R_{-1}, R_{1}, \ldots, N R_{N}\right)
$$

and

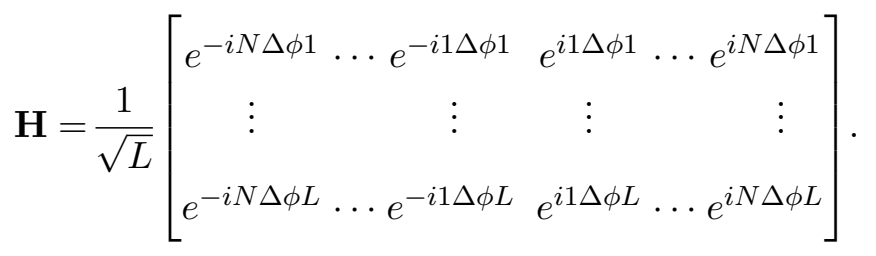

Note that the mode coefficient $q_{0}$ is not included in any of these equations. In order to recover the mode vector $\mathbf{q}$ from the observations $\mathbf{g}$, the linear equation system needs to be solved by matrix inversion. $\mathbf{J}$ is a diagonal matrix is therefore trivial to invert. However, with $L>2 N$, the overall equation system 
is overdetermined, hence $\mathbf{H}$ needs to be inverted by its Moore-Penrose Pseudoinverse [27]

$$
\mathbf{H}^{\dagger}=(\underbrace{\mathbf{H}^{H} \mathbf{H}}_{\mathbf{I}_{2 N}})^{-1} \mathbf{H}^{H}=\mathbf{H}^{H},
$$

where $\mathbf{I}_{2 N}$ denotes the $2 N \times 2 N$ unity matrix. The least-squares estimate $\tilde{\mathbf{q}}$ of $\mathbf{q}$ based on the observations $\mathbf{g}$ is then given by

$$
\tilde{\mathbf{q}}=\mathbf{J}^{-1} \mathbf{H}^{H} \mathbf{g}=\underbrace{\mathbf{J}^{-1} \mathbf{H}^{H} \mathbf{H J}}_{\mathbf{I}_{2 N}} \mathbf{q}=\mathbf{q}
$$

and hence even an exact solution. This is because the chosen $\mathbf{g}$ has no components in the nullspace of $\mathbf{H}^{H}$ and is order-limited (aliasing free case). An alternative way to obtain the weights $q_{n}$ is given by equation (33).

In the next subsection, the recovery of the 0th mode is described.

\subsection{Recovery of the 0th Mode using an additional pressure sensor}

An additional pressure sensor located at $\phi=\phi_{p}$ is used as a means to recover the coefficient $q_{0}$, when all other sensors are TPG sensors.

Similar to the TPG in (38), the pressure at $\phi_{p}$ can be expressed as a function of $q_{n}$, where the HD $q(\phi)$ has still a limited order $N$. Combining (16) and (10) leads to

$$
p(\phi)=\sum_{n=-N}^{N} R_{n} \sqrt{2 \pi} e^{i n \phi} q_{n} .
$$

This sum can also be expressed using linear algebra:

$$
p\left(\phi_{p}\right)=\left[\begin{array}{ll}
\sqrt{2 \pi} R_{0} & \mathbf{b}
\end{array}\right]\left[\begin{array}{c}
q_{0} \\
q_{-N} \\
\vdots \\
q_{-1} \\
q_{1} \\
\vdots \\
q_{N}
\end{array}\right]
$$


with

$$
\mathbf{b}=\sqrt{2 \pi}\left[R_{-N} e^{-i N \phi_{p}} \cdots R_{-1} e^{-i 1 \phi_{p}} \quad R_{1} e^{i 1 \phi_{p}} \cdots R_{N} e^{i N \phi_{p}}\right] .
$$

With the recovery of all $2 N+1$ coefficients but $q_{0}$ from the TPG observation, the only unknown in equation (45) is $q_{0}$. Using the result for $\tilde{\mathbf{q}}$, the solution for $\tilde{q}_{0}$ is then given as

$$
\tilde{q}_{0}=\frac{1}{\sqrt{2 \pi} R_{0}}\left[p\left(\phi_{p}\right)-\mathbf{b} \tilde{\mathbf{q}}\right]=q_{0}
$$

which is also an exact solution when (38) is satisfied.

An equivalent formulation of the same result is to comprise the full array observation $\mathbf{g}$ and $p\left(\phi_{p}\right)$ in an extended equation system

$$
\begin{gathered}
\mathbf{g}_{e}=\left[\begin{array}{l}
p \\
\mathbf{g}
\end{array}\right]=\mathbf{Y}\left[\begin{array}{l}
q_{0} \\
\mathbf{q}
\end{array}\right]=\mathbf{Y} \mathbf{q}_{e} \\
\mathbf{Y}=\left[\begin{array}{cc}
\sqrt{2 \pi} R_{0} & \mathbf{b} \\
\mathbf{0} & \mathbf{H J}
\end{array}\right]_{(L+1) \times(2 N+1)}, \quad \mathbf{0}=\left[\begin{array}{c}
0 \\
\vdots \\
0
\end{array}\right]_{L \times 1} .
\end{gathered}
$$

The system matrix $\mathbf{Y}$ is a composition of different matrices and vectors, which clearly limits the contribution of the $0 t h$ mode to the observed pressure $p$. The subscript $e$ serves to distinguish between the original and the extended vectors. In the light of the results found in (42), (43) and (46), the Moore-Penrose Pseudoinverse of $\mathbf{Y}$ is of the form

$$
\mathbf{Y}^{\dagger}=\left[\begin{array}{cc}
\frac{1}{\sqrt{2 \pi} R_{0}} & \mathbf{v} \\
\mathbf{0} & \mathbf{J}^{-1} \mathbf{H}^{H}
\end{array}\right]
$$

which has been confirmed by numerical results. As already implied by eq. (48), it is clear that $p$ in $\mathrm{g}_{e}$ is not used to recover any coefficients other than $q_{0}$, because the contribution of $q_{0}$ to $p$ cannot be compensated for, in general, by a linear combination of the elements of $\mathbf{g}$.

In fact, the analytic expression of the vector $\mathbf{v}$ can be derived from the findings above. From (43), 
(46) and (48) it is evident that $\mathbf{v}$ needs to satisfy

$$
\begin{aligned}
\frac{-1}{\sqrt{2 \pi} R_{0}} \mathbf{b q} & =\frac{-1}{\sqrt{2 \pi} R_{0}} \mathbf{b J}^{-1} \mathbf{H}^{H} \mathbf{g}=\mathbf{v g} \\
\Longrightarrow \mathbf{v} & =\frac{-1}{\sqrt{2 \pi} R_{0}} \mathbf{b} \mathbf{J}^{-1} \mathbf{H}^{H}
\end{aligned}
$$

The $L$ components of $\mathbf{v}$ are therefore

$$
v_{l}=\frac{i r_{V}}{L R_{0}} \sum_{\substack{n=-N \\ n \neq 0}}^{N} \frac{1}{\sqrt{2 \pi} n} e^{i n \phi_{P}} e^{-i n \Delta \phi l}, l \in[1, \ldots, L] .
$$

The expression in (49) defines $\mathbf{v}$ uniquely, since $\mathbf{g}$ can be any element of an $L$ dimensional vector space.

The final result for all recovered mode coefficients is

$$
\tilde{\mathbf{q}}_{e}=\mathbf{Y}^{\dagger} \mathbf{g}_{e}
$$

It has been shown that, provided the HD $q(\phi)$ defining the sound field leading to the observation of $\mathbf{g}_{e}$ does not contain any modes $a_{n}(\phi),|n|>N$, the application of the Moore-Penrose Pseudoinverse $\mathbf{Y}^{\dagger}$ leads to an exact solution for $\tilde{\mathbf{q}}_{e}$, and all coefficients $q_{n}$, including the 0th order coefficient $q_{0}$, are recovered correctly.

Unfortunately, sound fields are typically not order-limited. The aliasing pattern for an array observing the TPG only has already been presented in subsection 3.1; the consequences of aliasing on the recovery of $q_{0}$ are investigated in the following subsection.

\subsection{Robustness of the 0th Order}

It has been shown in the previous subsection that the mode coefficient $q_{0}$ can be recovered successfully if the condition

$$
q_{n}=0, \forall|n|>N
$$

is satisfied (compare to eq. (46)). This subsection deals with the recovery of the coefficients $q_{n}$ in the presence of spatial aliasing.

For a HD of infinite order, equation (36) describes exactly how the elements of $\tilde{\mathbf{q}}$ are distorted, yet 
it does not predict how $\tilde{q}_{0}$ is affected. Evidently, from equation (46) it can be seen that a recovered coefficient vector $\tilde{\mathbf{q}}$ corrupted by aliasing also results in an inaccurate recovery of $q_{0}$, namely

$$
\tilde{q}_{0} \neq q_{0}
$$

As a consequence, $q_{0}$ can only be recovered accurately if (53) holds. Whenever this condition is not satisfied, not only are the observed coefficients $\tilde{\mathbf{q}}$ degraded in accordance with the scheme given by orthogonality matrix $A_{m, n}$, but $q_{0}$ is subject to aliasing.

In conclusion, the aliasing pattern of the array with the additional pressure sensor can only partially be described by $A_{m, n}$ as the corruption of the 0th order mode is not accounted for. Fig. 5 qualitatively indicates the resulting orthogonality matrix $\tilde{A}_{m, n}$ obtained after combining $A_{m, n}$ with the effect that corrupted higher orders $\tilde{q}_{n}, 0<|n| \leq N$ have on the 0th order.

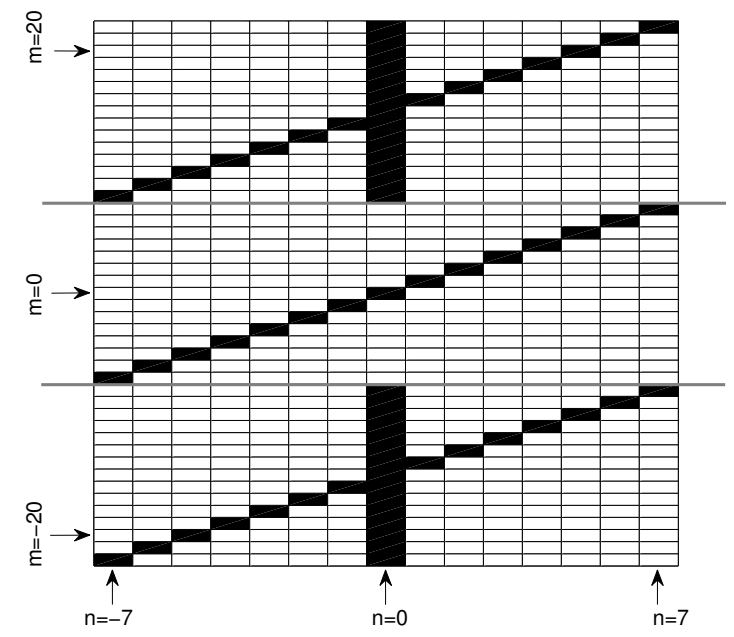

Figure 5: Schematic visualisation of the new orthogonality matrix $\tilde{A}_{m, n}$ for a circular array with $L=15 \mathrm{TPG}$ sensors and one pressure sensor. The two light grey lines indicate the mode range $( \pm N)$ of the array.

When comparing the aliasing patterns in Fig. 4 and Fig. 5, it can be seen that the TPG sensor array with an additional pressure sensor is more vulnerable to aliasing than a system based only on pressure sensors.

\subsection{HD Coefficients of the Sound Field of a Plane Wave}

The HD of a single plane wave of unity magnitude travelling in the direction $\phi_{i}$ is given by

$$
q(\phi)=\delta\left(\phi-\phi_{i}\right)
$$


This leads to the following TPG

$$
g(\phi)=\frac{1}{r_{V}} \sum_{n=-\infty}^{\infty} i n R_{n} e^{i n \phi} e^{-i n \phi_{i}}
$$

The Fourier coefficients of the HD given in (54) are

$$
q_{n}=\int_{0}^{2 \pi} q(\phi) \frac{e^{-i n \phi}}{\sqrt{2 \pi}} d \phi=\frac{1}{\sqrt{2 \pi}} e^{-i n \phi_{i}}
$$

For comparison with the work of Williams [1], the following equation describes the relation between the coefficients used in this work and the helical wave coefficients $C_{n}$ in Williams' work (compare [1], Section 4.3, pp. $121 \mathrm{ff})$.

$$
q_{n}=\frac{i^{-n}}{(2 \pi)^{\frac{3}{2}}} C_{n}
$$

Equation (56) shows that for a single plane wave, the magnitude of all coefficients is $\frac{1}{\sqrt{2 \pi}}$.

\subsection{Example of Mode Recovery With Spatial Aliasing}

Fig. 6 shows the mode recovery performance of an array of $L=15$ TPG sensors and one pressure sensor. The simulated sound field has a limited order $N_{s} \geq N\left(q_{n}=0, \forall|n|>N_{s}\right)$ and the coefficients $q_{n}$ are specified by (56). The wave field is an approximation of a plane wave within a radius $r_{P W} \approx \frac{N_{s}}{k}$ around the origin $[28,29]$. The theoretical magnitude of the HD coefficients is indicated by a dashed
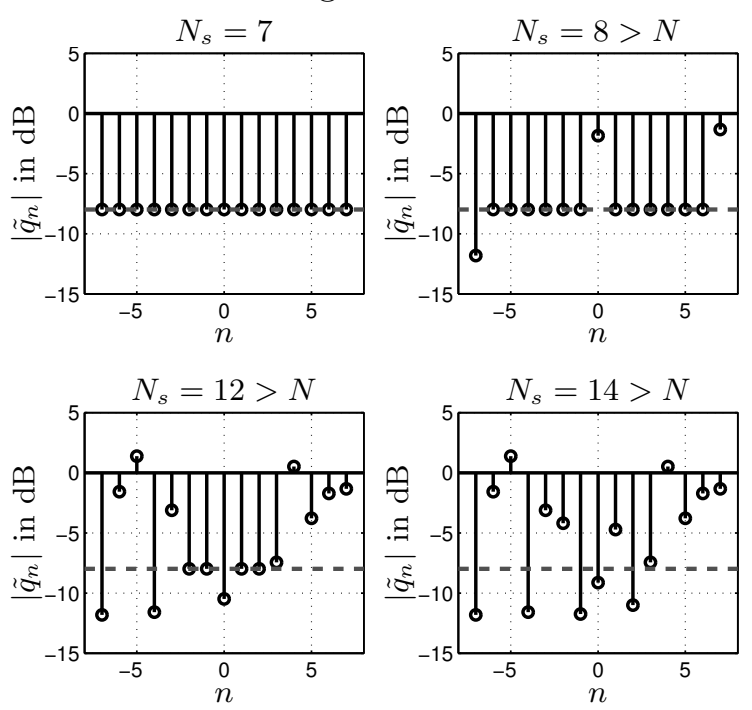

Figure 6: Mode recovery performance of a circular array composed of $L=15$ TPG sensors and one pressure sensor with $r_{V}=0.1 \mathrm{~m}$. The incoming sound field has a frequency $f=5 \mathrm{kHz}$ and the results are shown for $N_{s}=[7,8,12,14]$. The dashed grey line indicates the theoretical values for the magnitude of the HD coefficients, while the black stems display the recovered coefficients' magnitude. 
grey line in all four graphs. The top left graph shows that for $N_{s}=7=N$, the observed mode coefficients are recovered perfectly. The other three graphs however confirm the findings of subsection 3.4 and the aliasing scheme shown in Fig. 5. The graph for $N_{s}=8$ shows that $q_{-7}, q_{7}$ and $q_{0}$ are affected by aliasing. For $N_{s}=12$, only the coefficients $q_{n}, n \in[-2,-1,1,2]$ are still recovered correctly, while for $N_{s}=14$ all coefficients are distorted by aliasing. This is consistent with the pattern given in Fig. 5. The simulated sound field $\left(\phi_{i}=0, f=5 \mathrm{kHz}, c=343 \frac{\mathrm{m}}{\mathrm{s}}, N_{s}=8\right)$ and its reproduction based

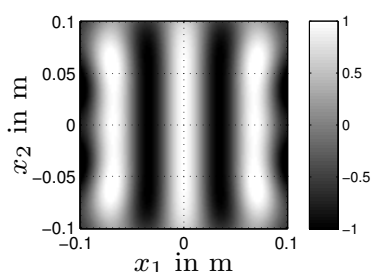

(a) Original Sound Field

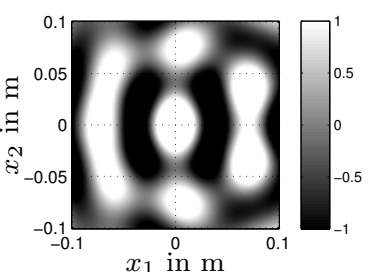

(b) Reproduced Sound Field

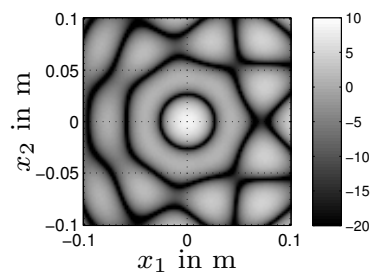

(c) Absolute Amplitude Error in $\mathrm{dB}$

Figure 7: Visualisation of the sound field with $\phi_{i}=0, f=5 \mathrm{kHz}, c=343 \frac{\mathrm{m}}{\mathrm{s}}$, limited to order $N_{s}=8$, (a) original sound field, (b) sound field as reproduced from the observation of $\tilde{q}_{n}$, using the microphone array described above, (c) absolute amplitude error of the reproduced field, given in $\mathrm{dB}$.

on the observed information $\tilde{\mathbf{q}}$ can be seen in Fig. 7 for $N_{s}=8$. Despite the order limitation, it still resembles that of a plane wave. The middle picture shows that the shape of the incoming wave fronts are significantly deformed, even though only three modes have been corrupted by aliasing (compare to Fig. 5 and Fig. 6). Especially the influence of the corrupted 0th order mode is clearly visible in the form of the concentric circle around the origin in the bottom graphic depicting the difference between the original and the reconstructed sound field.

\section{Simulations and Quantitative Error Analysis}

As it has been argued in subsection 2.4, it can be expected that TPG sensor arrays are more robust against measurement noise than pressure sensor arrays. This is due to the considerable difference in the magnitude of the eigenvalues and is most significant at low frequencies. At the same time, the findings in subsections 3.1 and 3.4 indicate that the TPG sensor design is more vulnerable to spatial 
aliasing than conventional designs. In order to confirm both hypotheses, a vast number of simulated measurements of an arbitrary sound field specified by (56) with different $\phi_{i}$ have been performed in MATLAB. These involve a simulated TPG array with $L_{G}=15$ uniformly spaced TPG sensors plus an additional pressure sensor at $\phi_{p}=\frac{\pi}{6}$, yielding a total amount of 16 sensors. The simulated conventional array consists of $L_{P}=16$ uniformly spaced pressure sensors. That leaves both arrays with the same number of sensors overall and both had a radius of $r_{V}=0.1 \mathrm{~m}$. They were both set to recover all the HD coefficients $q_{n}$ for $|n| \leq 7$. The signals observed at the various array sensors have been computed using the analytical solutions in equation (38) for the TPG and (44) for the pressure, both accounting for orders up to $|n|=N_{s}=40$. Note that this will lead to spatial aliasing with the given array at high frequencies. The needed HD coefficients $q_{n}$ are specified by equation (56).

The following three subsections describe the noise model, the regularisation of the inverse matrix $\mathbf{Y}^{\dagger}$ and the quality measure used in the simulations. Subsection 4.4 then presents the quantitative performance results for both array types.

\subsection{The Noise Model}

In order to compare the robustness of the two different arrays to measurement noise, the simulated measured signals were corrupted with a noise signal $d$. The noise in (ultrasonic) transducers has been identified to originate predominantly from the electronic circuitry [30,31]. Electrical noise consists mainly of four components:

- thermal noise due to thermal agitation of charges in conductors [32],

- shot noise (as it typically occurs in semiconductors and vacuum tubes),

- $1 / f$ noise (e.g. caused by impedance fluctuations in combination with DC currents occurring in the sensor's internal impedance converters) and

- flicker noise [33] (e.g. as it occurs in MOSFETs, which are frequently used as internal impedance converters).

The last three kinds of noise are decaying in level towards higher frequencies, where they are covered by the thermal noise, which is spectrally white. Assuming transducers with carefully designed impedance converters in combination with high quality signal pre amplifiers, it is deemed valid to model the overall noise with a white spectrum. Additionally, it is assumed that the conversion principle between 
the acoustic and the electrical quantities as well as the thermal agitation of the molecules in the fluid before the transducer are negligible compared to the electrical noise. Therefore, the overall noise can be modelled as a random signal with the same constant average magnitude at all frequencies for both types of transducers. For an individual sensor, the additive noise component is then specified by

$$
d=\sigma_{n} e^{i 2 \pi \mu}
$$

where $\mu$ is a uniformly distributed random variable on the interval $[0,1]$ and $\sigma_{n}$ is the standard deviation of the noise. To further randomise the nature of the noise, $\sigma_{n}$ was chosen to also be a random variable

$$
\sigma_{n}=1 \cdot 10^{-60 / 20} \xi
$$

where $\xi$ is a normally distributed random variable with unit variance. The average magnitude of the noise is then set to $60 \mathrm{~dB}$ below that of a pressure sensor's output signal in the undisturbed field of a plane wave with unit amplitude. Note that on a rigid cylinder, the average magnitude of the measured signals for either type of sensors depends on the sensor's position, the frequency and the direction of travel of the incoming plane wave.

The final signal model for the two sensors is then given as follows:

$$
\begin{aligned}
& \tilde{g}\left(\phi_{s}\right)=g\left(\phi_{s}\right)+d, \\
& \tilde{p}\left(\phi_{s}\right)=p\left(\phi_{s}\right)+d .
\end{aligned}
$$

\subsection{Regularisation Against Ill-Conditioning}

The recovery of the coefficients $q_{n}$ from noisy measurements poses an ill-conditioned problem at low frequencies due to the excessive gains applied as a consequence of the inversion of the eigenvalues $\lambda_{n}$

and $\nu_{n}$. Therefore, these simulations require a regularised Moore-Penrose Pseudoinverse $\mathbf{Y}_{R}^{\dagger}$ to solve the inverse problem. In this work, a Tikhonov regularisation [34] has been applied. The regularised pseudoinverse is then defined as

$$
\mathbf{Y}_{R}^{\dagger}=\left(\mathbf{Y}^{H} \mathbf{Y}+\Gamma^{T} \Gamma\right)^{-1} \mathbf{Y}^{H}
$$

where $\Gamma=\beta \mathbf{I}$. The matrix $\mathbf{I}$ is an $\left(L_{G}+1\right) \times\left(L_{G}+1\right)$ identity matrix for the TPG sensor array and an $L_{P} \times L_{P}$ identity matrix for the pressure sensor array, respectively. The value for $\beta$ was chosen 
empirically and set to $\sqrt{10^{\frac{-50}{20}}}$, which is equivalent to impose a maximum bound of $50 \mathrm{~dB}$ to the largest eigenvalue of the inverse matrix. The coefficients $\tilde{q}_{n, R}$ are then calculated from the noisy array observation $\tilde{\mathbf{g}}_{e}$ using

$$
\tilde{\mathbf{q}}_{e, R}=\mathbf{Y}_{R}^{\dagger} \tilde{\mathbf{g}}_{e}
$$

The elements of $\tilde{\mathbf{g}}_{e}$ are computed for orders up to $|n|=40$ and contain noise. The coefficients in $\tilde{\mathbf{q}}_{e, R}$ are thus corrupted by measurement noise, regularisation and spatial aliasing effects.

\subsection{The Quality Measure}

The accuracy of the recovered coefficients $\tilde{q}_{n, R}$ can be evaluated based on the energy of the error between the original HD $q(\phi)$ and the recovered $\mathrm{HD} \tilde{q}(\phi)$. The error-to-signal-ratio in $\mathrm{dB}$ for a given frequency is then given by

$$
\mathrm{ESR}=10 \cdot \log _{10}\left(\frac{\sum_{n=-N}^{N}\left|\tilde{q}_{n, R}-q_{n}\right|^{2}}{\sum_{n=-N}^{N}\left|q_{n}\right|^{2}}\right) \mathrm{dB} .
$$

\subsection{Simulation Results}

The ESR is used to compare the performance of the TPG array and the pressure array in a statistical analysis for a finite number of frequencies in the range between $100 \mathrm{~Hz}$ and $10 \mathrm{kHz}$ with a step size of $10 \mathrm{~Hz}$. For each considered frequency $f, 100$ simulated measurements were conducted: i.e. 10 iterations each for 10 randomly selected approximated plane wave fields (see subsection 3.6). This allows for a good approximation of the noise model and the aliasing performance is not biased by the choice of specific directions of incidence for the incoming waves.

Fig. 8 shows the result of the analysis, where the black line depicts the performance of the TPG sensor array and the light grey line the performance of the pressure sensor array. The plots clearly show the effects of spatial aliasing and noise. While the error at high frequencies is dominated by the effect of spatial aliasing, the effect of transducer noise is dominant at low frequencies.

As suggested by the findings in subsections 3.1 and 3.4, the error due to spatial aliasing at high frequencies is higher with the TPG sensor array than with the pressure sensor array. Above $f=$ $1.8 \mathrm{kHz}$ the conventional array clearly outperforms the TPG array with the ESR reaching values below $-30 \mathrm{~dB}$, yet it should be mentioned that the conventional array with $L_{P}=16>2 N+1$ sensors is more robust to aliasing from higher orders than a conventional array with $L_{P}=15=2 N+1$ 


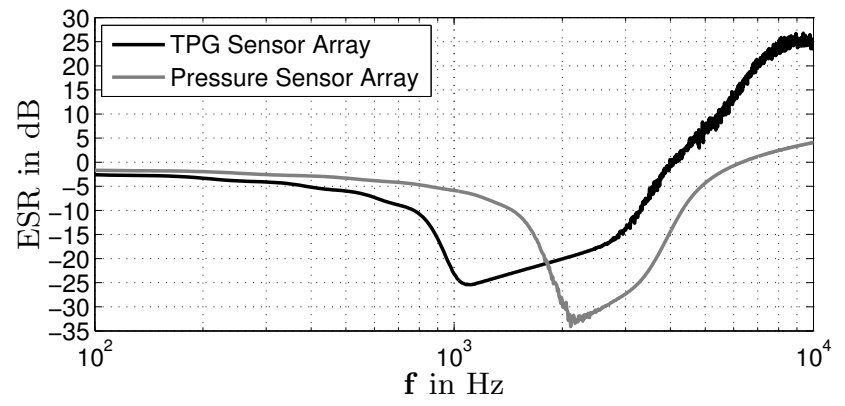

Figure 8: Plots of the average ESR of the recovered coefficients $\tilde{q}_{n, R}$ from a simulated TPG sensor array measurement (black line) and from a simulated pressure sensor array measurement (light grey line) within the frequency range from $100 \mathrm{~Hz}$ to $10 \mathrm{kHz}$.

sensors.

At lower frequencies, the performance of the TPG sensor array is significantly better than that of the conventional array.

Judging by the width of the respective frequency band in which the two arrays' ESR falls below $-15 \mathrm{~dB}$, both arrays achieve a band that is around $2.3 \mathrm{kHz}$ wide. However, w.r.t. the lowest ESR value, the conventional array outperforms the TPG sensor array by around $6.5 \mathrm{~dB}$.

It is worth noticing that the optimum band of the TPG sensor array is shifted towards lower frequencies. This can be achieved with a conventional array by increasing its radius, which shifts the curves of the eigenvalues in Fig. 2 and Fig. 3 towards lower frequencies due to the change of the argument in the radial functions. However, comparing a TPG sensor array with a conventional array of the same radius, it is clear that the former performs better at low frequencies. Therefore, considering audio applications, the two the arrays combined on one structure would extend the usable bandwidth of the TPG sensor array by $45 \%$ or that of the conventional array by $33 \%$, respectively. This would gain a substantially increased accuracy within a frequency band where the human ear is very sensitive (compare to the curves of equivalent loudness in ISO 226) and which is very important for localisation (due to interaural time difference and spectral cues from head and torso reflections [35]).

The following simulation example visualises the effect of the improvement over conventional designs at low frequencies.

\subsection{An Example}

With $L_{P}=16$ pressure sensors and $L_{G}=15$ TPG sensors plus the additional pressure sensor, the respective arrays allow for the recovery of the coefficients $q_{n},|n| \leq 7$. From Fig. 2 and Fig. 3 it can 


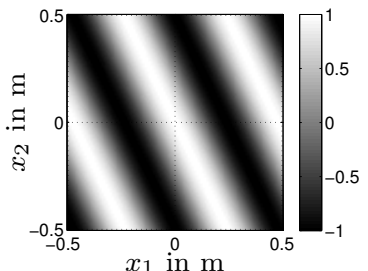

(a)

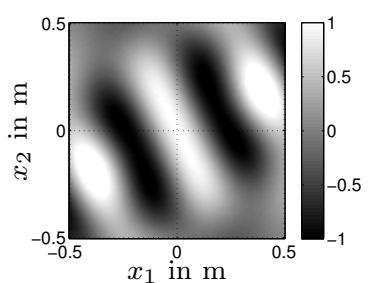

(c)

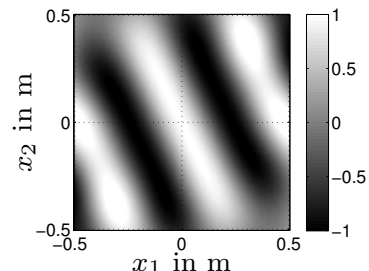

(b)

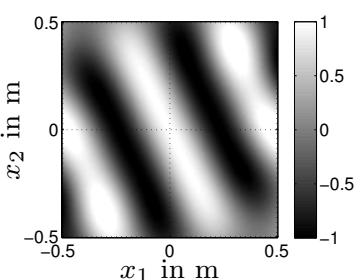

(d)

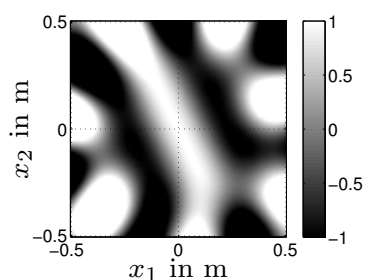

(e)

Figure 9: Visualisation of a plane wave approximation with $\phi_{i}=\frac{\pi}{7}, f=800 \mathrm{~Hz}, c=343 \frac{\mathrm{m}}{\mathrm{s}}$, limited to order $N_{s}=15$, (a) original sound field, (b) synthesis based on TPG array measurement with $\beta=\sqrt{10^{\frac{-50}{20}}}$, (c) synthesis based on pressure array measurement with $\beta=\sqrt{10^{\frac{-50}{20}}}$, (d) synthesis based on TPG array measurement with $\beta=0$, (e) synthesis based on pressure array measurement with $\beta=0$.

be seen that the gain applied to the array observation for the recovery of $q_{-7}$ and $q_{7}$ at a frequency of $f=800 \mathrm{~Hz}$ is about $35 \mathrm{~dB}$ for the TPG array and about $70 \mathrm{~dB}$ for the conventional array. With a given SNR of $60 \mathrm{~dB}$, it can be expected that the noise is going to affect the measurement of the conventional array considerably more than that of the TPG array.

Fig. 9 shows the reconstruction of a plane wave approximation $\left(\phi_{i}=\frac{\pi}{7}, f=800 \mathrm{~Hz}, c=\right.$ $\left.343 \frac{\mathrm{m}}{\mathrm{s}}, N_{s}=15\right)$ on the basis of the coefficients $\tilde{\mathbf{q}}$ recovered from a TGP sensor array and from a pressure sensor array for the cases of $\beta=\sqrt{10^{\frac{-50}{20}}}$ and $\beta=0$. It shows that the synthesis based on the measurement of the TPG array with and without regularisation is only marginally different from the original sound field. With the pressure array, regularisation leads to a suppression of higher orders, resulting in a reduced size of the region of accurate reconstruction $[28,29]$ and without regularisation, the noise leads to a spatially distorted synthesis outside the region where 6th order contributions become significant. This result supports the results in subsection 4.4 that TPG sensor arrays are more 
robust against transducer noise than conventional arrays.

\section{Conclusions}

The performance of a circular microphone array composed of TPG sensors has been investigated by means of theoretical analysis and numerical simulations. The integral operator describing the sound field model has been introduced and decomposed by means of the eigenvalue decomposition. This allows for an entirely separate analysis of transducer noise induced errors and aliasing effects, since both can be associated with different components of the EVD.

It has been shown that, at low frequencies, a TPG sensor array is more robust against transducer noise than conventional arrays, while, at high frequencies, it is significantly more vulnerable to spatial aliasing. In the simulations presented in this work, the lowest achievable ESR of the TPG sensor array is surpassed by that of a comparable conventional array by approximately $6.5 \mathrm{~dB}$. Furthermore, it was found that the usable optimum frequency band is not extended by the new design, but it is shifted towards lower frequencies. Finally, even though it has been found that this new design can potentially gain on former designs in terms of transducer noise, this is only true under the condition that both transducer types are of equal quality. The costs of a gradient sensor that matches the quality (w.r.t. noise, symmetry of its directivity, etc.) of a state-of-the-art pressure sensor are however expected to be considerably higher. It is therefore possible that what the TPG sensor array gains in robustness at low frequencies is not enough to compensate for the generally higher transducer noise.

It has also been shown that for the recovery of the 0th mode, at least one pressure sensor needs to be added to the array. This leads to a change in the spatial aliasing scheme of the TPG array, making the 0th mode particularly vulnerable to aliasing.

\section{Acknowledgement}

We would like to thank Peter Craven, Chris Travis and Malcolm Law for the inspiration that lead to this work.

This work has been partially funded by the Royal Academy of Engineering and by the Engineering and Physical Sciences Research Council. 


\section{References}

[1] E. G. Williams, Fourier Acoustic: Sound Radiation and Nearfield Acoustical Holography. Academic Press, 1999.

[2] B. D. V. Veen and K. M. Buckley, "Beamforming: A versatile approach to spatial filtering," IEEE ASSP Magazine, vol. 5, no. 2, pp. 4-24, April 1988.

[3] H. Krim and M. Viberg, "Two decades of array signal processing research: the parametric approach," Signal Processing Magazine, IEEE, vol. 13, no. 4, pp. 67-94, Jul 1996.

[4] A. D. Blumlein, "Improvements in and relating to sound-transmission, sound-recording and sound-reproducing systems," Great Britain Patent 394 325, 1931.

[5] —_, "Improvements in and relating to electrical sound transmission systems," Great Britain Patent 456,444, 1935.

[6] P. G. Craven and M. A. Gerzon, "Coincident microphone simulation covering three dimensional space and yielding various directional outputs," USA Patent US4042 779 A, 1974.

[7] J. Meyer and G. W. Elko, "A highly scalable spherical microphone array based on an orthonormal decomposition of the soundfield." in ICASSP. IEEE, 2002, pp. 1781-1784.

[8] B. Rafaely, "Analysis and design of spherical microphone arrays," IEEE Transactions on Speech and Audio Processing, vol. 13, no. 1, pp. 135-143, January 2005.

[9] Y. Li and R. Duraiswami, "Flexible and optimal design of spherical microphone arrays for beamforming," IEEE Transactions on Speech and Audio Processing, vol. 15, no. 2, pp. 702-714, 2007.

[10] D. N. Zotkin, R. Duraiswami, and N. A. Gumerov, "Plane-wave decomposition of acoustical scenes via spherical and cylindrical microphone arrays," IEEE Transactions on Audio, Speech, and Language Processing, vol. 18, no. 1, pp. 2-16, January 2010.

[11] B. Rafaely, "Plane-wave decomposition of the sound field on a sphere by spherical convolution," Journal of the Acoustical Society of America, vol. 4, pp. 2149-2157, October 2004.

[12] M. Park and B. Rafaely, "Sound-field analysis by plane-wave decomposition using spherical microphone array," Journal of the Acoustical Society of America, vol. 118, pp. 3094-3103, November 2005. 
[13] B. Rafaely, B. Weiss, and E. Bachmat, "Spatial aliasing in spherical microphone arrays," IEEE Transactions on Signal Processing, vol. 55, pp. 1003-1010, 2007.

[14] M. A. Poletti, "Three-dimensional surround sound systems based on spherical harmonics," Journal of the Audio Engineering Society, vol. 53, no. 11, pp. 1004-1025, November 2005.

[15] F. M. Fazi and P. A. Nelson, "Nonuniqueness of the solution of the sound field reproduction problem with boundary pressure control," Acta Acustica united with Acustica, vol. 98, no. 1, pp. 1-14, January 2012.

[16] J. Meyer, "Beamforming for a circular microphone array mounted on spherically shaped objects," The Journal of the Acoustical Society of America, vol. 109, no. 1, pp. 185-193, Jan. 2001.

[17] H. Teutsch and W. Kellermann, "Acoustic source detection and localization based on wavefield decomposition using circular microphone arrays," Journal of the Acoustical Society of America, vol. 120, no. 5, pp. 2724-2736, November 2006.

[18] M. Kleider, B. Rafaely, B. Weiss, and E. Bachmat, "Golden-ratio sampling for scanning circular microphone arrays," IEEE Transactions on Audio, Speech, and Language Processing, vol. 18, pp. 2091-2098, 2010.

[19] J. Meyer and G. W. Elko, "Spherical harmonic modal beamforming for an augmented circular microphone array." in ICASSP. IEEE, 2008, pp. 5280-5283.

[20] M. A. Poletti, "Effect of noise and transducer variability on the performance of circular microphone arrays," J. Audio Eng. Soc, vol. 53, no. 5, pp. 371-384, 2005.

[21] P. G. Craven, M. J. Law, and C. Travis, "Microphone arrays using tangential velocity sensors," in Ambisonics Symposium, June 2009.

[22] F.-M. Hoffmann and F. Fazi, "Circular microphone array with tangential pressure gradient sensors," in Hands-free Speech Communication and Microphone Arrays (HSCMA), 2014 4th Joint Workshop on, May 2014, pp. 97-101.

[23] D. Colton and R. Kress, Inverse Acoustic and Electromagnetic Scattering Theory, Series 93 Applied Mathematical Sciences. Springer, 1998. 
[24] F. M. Fazi, "Sound field reproduction," Ph.D. dissertation, University of Southampton, Faculty of Engineering, Science and Mathematics, Institute of Sound and Vibration Research, 2010.

[25] F. M. Fazi, M. Noisternig, and O. Warusfel, "Representation of sound fields for audio recording and reproduction," in Acoustics 2012: 11ème Congrès Français d'Acoustique. Annual meeting of the Institute of Acoustics, Nantes, FR, 23 - 27 Apr 2012, 2012.

[26] S. Moreau, J. Daniel, and S. Bertet, "3d sound field recording with higher order ambisonics objective measurements and validation of a 4th order spherical microphone," in Preprints of the 120th AES Convention, 2006.

[27] S. O. Haykin, Adaptive filter theory, 4th ed. Upper Saddle River, NJ: Prentice Hall, 2002.

[28] D. B. Ward and T. D. Abhayapala, "Reproduction of a plane-wave sound field using an array of loudspeakers," IEEE Transactions on Speech and Audio Processing, vol. 9, no. 6, pp. 697-707, September 2001.

[29] J. Ahrens, Analytic Methods of Sound Field Synthesis, ser. T-Labs Series in Telecommunication Services. Springer, 2012.

[30] G. Hayward, R. Banks, and L. Russell, "A model for low noise design of ultrasonic transducers," in Ultrasonics Symposium, 1995. Proceedings., 1995 IEEE, vol. 2, Nov 1995, pp. 971-974 vol.2.

[31] C. Oakley, "Calculation of ultrasonic transducer signal-to-noise ratios using the klm model," Ultrasonics, Ferroelectrics, and Frequency Control, IEEE Transactions on, vol. 44, no. 5, pp. 1018-1026, Sept 1997.

[32] H. Nyquist, "Thermal agitation of electric charge in conductors," Phys. Rev., vol. 32, pp. 110-113, Jul 1928.

[33] W. Schottky, "Small-shot effect and flicker effect," Phys. Rev., vol. 28, pp. 74-103, Jul 1926.

[34] S. Mallat, A Wavelet Tour of Signal Processing, Third Edition: The Sparse Way, 3rd ed. Academic Press, 2008.

[35] J. Blauert, Spatial Hearing : The Psychophysics of Human Sound Localization. Cambridge, Mass. MIT Press, 1997. 